\title{
Numerical method for computing Maass cusp forms on triply punctured two-sphere
}

\begin{abstract}
A quantum mechanical system on a punctured surface modeled on hyperbolic space has always been an important subject of research in mathematics and physics. This corresponding quantum system is governed by the Schrödinger equation whose solutions are the Maass waveforms. Spectral studies on these Maass waveforms are known to contain both continuous and discrete eigenvalues. The discrete eigenfunctions are usually called the Maass Cusp Forms (MCF) where their discrete eigenvalues are not known analytically. We introduce a numerical method based on Hejhal and Then algorithm using GridMathematica for computing MCF on a punctured surface with three cusps namely the triply punctured twosphere. We also report on a pullback algorithm for the punctured surface and a point locater algorithm to facilitate the complete pullback which are essential parts of the main algorithm.
\end{abstract}

Keyword: Discrete eigenvalues; Maass cusp forms; Numerical method 